\section{$\underset{\substack{\text { hommes } \\ \text { \& migrations }}}{ }$}

\section{Hommes \& migrations}

Revue française de référence sur les dynamiques

migratoires

$1326 \mid 2019$

Londres et ses migrations

\title{
Le rôle de l'intégration sociale dans les politiques mises en œuvre par l'Autorité du Grand Londres entre 2016 et 2019
}

\section{Jacqueline Broadhead}

Traducteur : Alexandre Pateau

\section{(2) OpenEdition \\ Journals}

\section{Édition électronique}

URL : https://journals.openedition.org/hommesmigrations/9641

DOI : 10.4000/hommesmigrations.9641

ISSN : 2262-3353

\section{Éditeur}

Musée national de l'histoire de l'immigration

\section{Édition imprimée}

Date de publication : 1 juillet 2019

Pagination : 49-57

ISBN : 978-2-919040-46-9

ISSN : $1142-852 X$

\section{Référence électronique}

Jacqueline Broadhead, « Le rôle de l'intégration sociale dans les politiques mises en œuvre par

l'Autorité du Grand Londres entre 2016 et $2019 »$, Hommes \& migrations [En ligne], 1326 | 2019, mis en ligne le 01 janvier 2022, consulté le 16 janvier 2022. URL : http://journals.openedition.org/

hommesmigrations/9641; DOI : https://doi.org/10.4000/hommesmigrations.9641 


\section{Le rôle de l'intégration sociale dans} les politiques mises en œuvre par l'Autorité du Grand Londres entre 2016 et 2019

\section{Jacqueline Broadhead,}

directrice du programme «Global Exchange on Migration and Diversity» («Échanges internationaux/globaux sur les migrations et la diversité») du Centre d'études sur les migrations, la politique et la société (Compas) de l'université d'Oxford.

La stratégie d'intégration sociale lancée par l'Autorité du Grand Londres (AGL) en 2016, et intitulée "All of Us» («Tous ensemble»), inaugure une nouvelle approche des politiques d'intégration dans la ville de Londres. Ce programme se concentre sur la répartition des responsabilités dans un environnement misant sur l'égalité, la participation et les liens sociaux. Par son approche sociale, il contribue à renforcer la pratique d'un leadership inclusif ancré dans l'environnement social londonien, en produisant des récits qui vont de pair avec sa volonté de faire évoluer les pratiques.

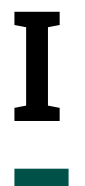

dentique en cela à d'autres villes britanniques, le rôle de Londres en matière d'intégration peut être décrit en reprenant les termes du groupe parlementaire multipartite (APPG) sur l'intégration sociale en 2017: "Une division mal définie des responsabilités en matière de politique d'intégration, qui se partage entre les départements et les agences du gouvernement central, et dont le manque de clarté est aggravé par l'absence d'une vision commune quant au rôle du gouvernement local dans ce domaine de la politique sociale. » Malgré le "tournant local» pris par la politique d'intégration ${ }^{\mathbf{1}}$ dans

1. Tiziana Caponio, Maren Borkert (dir.), The Local Dimension of Migration Policymaking, Amsterdam, Amsterdam University Press, 2010. 
le contexte de la décentralisation dans les années 2010, le système britannique est demeuré fortement centralisé, entravant le développement de certaines politiques d'intégration que l'on observe à la même époque dans d'autres villes européennes. Les questions d'intégration ont longtemps été considérées comme un problème secondaire par les autorités locales britanniques. L'absence d'une stratégie d'intégration à l'échelle nationale ou d'un mécanisme clairement définie n'a pas incité les gouvernements locaux à s'engager sur cette question.

Cela dit, la ville de Londres - la plus grande ville d'Angleterre et celle qui affiche des taux d'immigration bien plus élevés que n'importe quelle autre ville du pays ${ }^{2}$ - et l'Autorité du Grand Londres (AGL) en particulier se sont engagées dans le développement de politiques et de pratiques liées à l'intégration sociale et à l'immigration. Voici un aperçu des politiques d'intégration sociale de l'AGL depuis sa création en 2000. À travers une étude détaillée des structures politiques depuis 2016, cet article s'intéresse particulièrement à la façon dont l'AGL a choisi de se concentrer sur l'intégration sociale: un engagement en rupture importante par rapport aux administrations précédentes. Enfin, on identifie les opportunités et les défis que constitue l'effort porté sur les politiques sociales, et les différentes manières dont l'AGL pourrait chercher à développer cette approche à l'avenir.

\section{Placer l'intégration dans son contexte: l'action de l'AGL entre 2000 et 2016}

Créée en 2000, l'Autorité du Grand Londres (AGL) est placée sous la direction du maire de Londres, le plus ancien des «Metro Mayors», ces maires responsables de plusieurs villes, et dont le rôle a caractérisé le début d'une tendance accrue à la décentralisation des régions et des villes anglaises. Cependant, comme l'a exposé Mark Sandford en 2018, ses responsabilités et ses compétences sont uniques au sein du gouvernement local britannique. Opérant dans plus de 33 arrondissements londoniens, Sandford identifie des compétences clés de l'AGL dans les domaines liés au «transport, puis au logement, au maintien de l'ordre, au développement économique et à la rénovation urbain $e^{3} »$. Mais la mairie détient également un pouvoir de compétence générale (dérivé de la loi dite «Localism Act» de 2011) et un pouvoir d'action sur des thèmes transversaux définis par la loi de l'AGL de 1999 en lien avec «la gérance des objectifs en matière d'égalité à Londres ${ }^{4} \gg$. Ces nouvelles compétences ont permis la nomination d'un certain nombre de maires adjoints chargés de champs politiques particuliers, et parmi eux, le premier maire adjoint chargé de l'intégration sociale fut nommé en 2016. L'AGL réunit également le «Strategic Migration Partnership for London» («partenariat stratégique sur l'immigration de la ville de Londres», LSMP), mandaté pour agir en tant que "partenariat intersectoriel chargé de maintenir une vue d'ensemble stratégique de l'état de l'immigration à Londres». Le LSMP informe le ministère de l'Intérieur et le maire de Londres des principales questions et tendances en matière de politique d'immigration et d'intégration, et de leurs effets sur la croissance économique de Londres et son développement futur.

On peut donc affirmer à juste titre que, si l'intégration et l'immigration ne sont pas nécessairement identifiées comme des compétences essentielles de l'AGL, elles ont fait partie intégrante du mandat dès ses débuts, et se sont développées parallèlement aux tendances nationales en matière d'intégration et d'inclusion (quand bien même elles ne les reflétaient pas toujours).

Pendant le mandat du maire Ken Livingstone (de 2000 à 2008), l'action de l'AGL dans ce domaine faisait écho au programme principal du «New Labour» en matière d'égalité et, comme l'a montré Claire Worley en $2005^{5}$, un adjoint au maire chargé du maintien de l'ordre et de l'égalité fut nommé - ce lien direct entre sécurité communautaire et cohésion communautaire étant un thème structurel constant dans les politiques locales du gouvernement britannique - afin de mettre en œuvre le programme intitulé «Vision du maire sur les inégalités». Ce programme avait pour ambition de faire de l'AGL « un fer de lance en matière d'égalité et dans le combat contre les inégalités: remettre en question et éradiquer la discrimination,

2. $38 \%$ de sa population est née à l'étranger, un taux nettement plus élevé que la région qui arrive en deuxième position avec seulement $14 \%$, selon les chiffres publiés par l'Observatoire des migrations en 2018.

3. Mark Sandford, "The Greater London Authority », House of Commons Library Briefing, 2018. Url: https://researchbriefings parliament.uk/ResearchBriefing/Summary/SN05817.

4. Greater London Authority, «Race Equality Scheme 20052008 » 2005. Url: https://www.london.gov.uk/moderngov/Data/ Business $\% 20$ Management\%20and\%20Administration\%20 Committee/20050719/Agenda/8\%20Appendix\%20A\%20PDF.pdf.

5. Claire Worley, "It's not about Race. It's about the Community" : New Labour and "Community Cohesion" », in Critical Social Policy, vol. 25, n4, 2005, pp. 483-496. 
fournir des services adaptés et accessibles aux Londonien-nes et veiller à ce que le personnel de l'AGL reflète la diversité de la population de Londres ${ }^{6}$.» Une approche renforcée par la «commission des fonctionnaires et des parties prenantes pour l'égalité (Equalities Commission of Officers and Stakeholders)», qui s'est vue confier à la fois un rôle de développement et d'amélioration de la stratégie générale7 ${ }^{7}$. Il est évident que ces stratégies ont eu une influence sur la gestion des questions migratoires (notamment en ce qui concerne le conseil juridique pour les demandeurs d'asile, les réfugié-es et les femmes dont le statut d'immigrées les expose à un risque de violence domestique) et d'intégration. Toutefois, il faut préciser que cette approche s'est faite soit en visant les demandeurs d'asile et les réfugié-es, soit par le prisme plus large de l'égalité, plutôt qu'en appréhendant l'immigration ou l'intégration en tant que telle.

Le mandat du maire Boris Johnson (de 2008 à 2016) s'est d'abord focalisé sur les réfugié-es, en publiant le programme intitulé «London Enriched» («Enrichissons Londres») en 2009. Tout en affirmant que «certains de ces défis concernent d'autres immigrés venus du monde entier qui vivent et travaillent à Londres $^{8}$ », ce programme met clairement l'accent sur l'intégration des réfugié-es, marquant en cela un changement d'approche par rapport au programme de la mairie Livingstone', passant d'une focale plus large en matière d'égalité à une approche plus ciblée, et spécifiquement axée sur l'intégration. La stratégie elle-même définit l'intégration comme un «processus bilatéral exigeant un engagement positif à la fois de la part des réfugié-es et de la communauté établie. L'intégration englobe tous les aspects de la vie, qu'il s'agisse des domaines économique, social, culturel, civique ou politique ${ }^{10} »$. Cette approche est en phase avec la stratégie d'intégration du gouvernement national. Le London Strategic Migration Partnership (LSMP) et le Migration and Refugee Advisory Panel (groupe d'étude en charge de l'immigration et des réfugié-es, MRAP) sont les organes clés qui, dans le premier cas, «rassemblent des entités stratégiques régionales afin de planifier et de coordonner les prestations » et, dans le second, « reflètent les perspectives communautaires». Ben Gidley et Hiranthi Jayaweera ont montré l'intention d'élargir cette perspective, après sa première année de mise en œuvre, à d'autres groupes importants de migrants (ressortissant-es européen-nes, migration familiale, étudiants internationaux et travailleurs ou travailleuses immigré-es)"11. Une réflexion est engagée pour permettre d'adapter et élargir la philosophie du programme «Enrichissons Londres». Cette réflexion débouche sur une mise à jour de la stratégie en 2010. Elle élargit son champ d'application pour inclure à la fois les réfugié-es et les migrant-es. Le nouveau document identifie sept domaines politiques essentiels: la langue anglaise, le logement, l'emploi, les compétences et l'entreprise, la santé, la sécurité communautaire, la participation et le développement des enfants, des adolescents et des communautés.

\section{L'intégration sociale au sein de l'AGL après 2016}

L'élection du maire Sadiq Khan en 2016 a induit à la fois une continuité et un changement dans l'évolution du rôle de l'AGL, modifiant sa politique et sa pratique de l'intégration, ses priorités et sa vision. Le fait le plus frappant est peut-être que Khan, fidèle aux promesses de son programme, a nommé le premier maire adjoint chargé de l'intégration sociale, sur la recommandation de Sunder Katwala, Steve Ballinger et Will Somerville ${ }^{12}$, avec pour mission de s'inspirer des modèles américains de «bureaux en charge des questions migratoires» pour créer un "bureau en charge de la citoyenneté et de l'intégration». Le rapport développe une vision dans laquelle un nouveau maire adjoint «servirait de promoteur public du programme d'intégration», s'assurant que cette question soit constamment inscrite dans l'action de l'AGL, en étroit partenariat avec le LSMP, afin de promouvoir un «programme d'intégration et de citoyenneté favorisant la participation démocratique

\section{Ibid.}

7. Greater London Authority, «Race Equality Scheme 20052008 », op. cit.

8. Greater London Authority, "London Enriched. The Mayor's Strategy for Refugee Integration », 2009. Url: https://www.london. gov.uk/sites/default/files/gla_migrate_files_destination/ London\%20Enriched\%20The\%20Mayor\%27s\%20Strategy\%20 for\%20Refugee\%20Integration.pdf.

9. Donald Mcneill, «Livingstone's London : Left Politics and the World City », in Regional Studies, vol. 36, n 1, 2002, pp. 75-80.

10. Ibid.

11. Ben Gidley, Hiranthi Jayaweera, «An Evidence Base on Migration and Integration in London », in Centre on Migration, Policy and Society, University of Oxford, 2010. Url : https ://www. compas.ox.ac.uk/wp-content/uploads/PR-2010-Evidence_ Migration_London.pdf.

12. Sunder Katwala, Steve Ballinger, Will Somerville, « Making Citizenship Matter: Why London Needs an Office for Citizenship \& Integration », 2016. Url : http ://www.britishfuture.org/ publication/making-citizenship-matter/. 
et les échanges entre citoyen-nes et communautés à Londres - encourageant et défendant ainsi les nouveaux habitants de Londres ${ }^{13} \gg$.

Cette vision imprègne le rôle de l'AGL en matière d'intégration sous la gouvernance du maire Khan; un rôle dont on peut dire qu'il est constitué des éléments suivants: le leadership et les campagnes de communications de la mairie, notamment la campagne «London is Open» («Londres est ouverte»); la nomination d'un adjoint au maire chargé de l'intégration sociale et de l'élaboration ultérieure d'un programme d'intégration sociale favorisant l'égalité des chances; la prestation de services, notamment dans le cadre de l'«initiative sur la citoyenneté et l'intégration» du LSMP et du MRAP.

\section{Leadership du maire et communication stratégique}

Avant d'examiner le rôle de l'adjoint au maire et l'élaboration de la politique stratégique de l'AGL, il est important de considérer le rôle du maire. L'élection de Sadiq Khan en 2016 a été décrite par la BBC comme une «victoire symbolique à plusieurs égards», Khan devenant «l'un des premiers maires musulmans d'Europe, à une époque où les capitales européennes ont été mises à l'épreuve par les crises du terrorisme et le sort des réfugiés. [Khan] prend ses fonctions à l'issue d'une campagne entachée de tensions religieuses et raciales qui, si elles sont peut-être annonciatrices des problèmes à venir, pourraient aussi constituer une véritable chance $^{14}$ ». Khan lui-même, dans son discours d'entrée en fonction, a invoqué la victoire de "l'espoir sur la peur et l'unité sur la division ${ }^{15}$ ». Le nouveau maire fut élu en mai 2016. Moins d'un mois plus tard avait lieu le référendum sur le Brexit, auquel Khan répondait à l'été 2016 en lançant la campagne "London is Open» («Londres est ouverte»). Elle est décrite par London and Partners, l'agence de l'AGL ayant soutenu son développement, comme une «campagne majeure lancée en 2016 par le maire de Londres, Sadiq Khan, pour montrer que Londres reste unie et ouverte auxéchanges commerciaux. Cette campagne prouve au monde que Londres reste une ville internationale et entrepreneuriale, pleine de créativité et de possibles, tout en rassurant les plus d'un million de ressortissants étrangers vivant à Londres qu'ils et elles seront toujours les bienvenu-es et qu'aucune forme de discrimination ne sera tolérée $e^{16}$ ». Comme Els de Graauw et Irene Bloemraad l'ont montré en 2018 pour le contexte américain, l'un des points communs des stratégies locales en matière d'inclusion est de "faire valoir» - souvent dans une perspective économique - l'idée que «les immigré-es ont une importance économique actuelle et future pour les villes, les régions métropolitaines et les États, tout en encourageant leur engagement et leur intégration civiques ${ }^{17} »$. La campagne fut renforcée par un effort de promotion de la part du maire en matière de droits réciproques des citoyens de l'UE à Londres, Khan ayant déclaré dans sa première allocution post-référendum: «Je veux envoyer un message fort aux près d'un million d'Européen-nes vivant à Londres, qui apportent leur énorme contribution à notre ville - vous travaillez dur, vous payez vos impôts, vous enrichissez notre vie publique et culturelle. Vous êtes les bienvenus ${ }^{18}$.»

Ces campagnes illustrent les fonctions primordiales de la mairie en matière de leadership, dont certaines peuvent être considérées comme symboliques (et incarnées en quelque sorte par Khan lui-même et par la façon dont elles sont interprétées). Elles se retrouvent dans les décisions stratégiques en matière de communication prises au début du mandat du maire pour faire de Londres une ville «ouverte». La conceptualisation par Robin Hambleton d'un leadership axé sur l'inclusion montre clairement l'importance du leadership politique dans ce processus: même si on ne voit pas toujours le lien entre la communication faite par la mairie et la stratégie et la pratique mise en œuvre sur le terrain, ce type de gouvernance fournit sans nul doute une orientation et un cadre déterminants pour le développement de la stratégie de l'AGL en matière d'inclusion ${ }^{19}$.

13. Ibid.

14. Tim Donovan, «London Mayoral Election: How Sadiq Khan Won », in BBC News, 7 mai 2016. Url : https://www.bbc.co.uk/ news/election-2016-36230609.

15. Sadiq Khan, « Mayor of London Response to EU Referendum Result », 2016. Url : https://www.london.gov.uk/press-releases/ mayoral/mayor-of-london-response-to-eu-referendum-result.

16. London and Partners, «London is Open », 2016. Url : https :// www.londonandpartners.com/what-we-do/case-study-london-isopen.

17. Els de Graauw, Irene Bloemraad, « Working Together : Building Successful Policy and Program Partnerships for Immigrant Integration », in Journal on Migration and Human Security, vol. 5, $\mathrm{n}^{\circ} 1,2017$, pp. 105-123.

18. Sadiq Khan, op. cit.

19. Robin Hambleton, « Inclusive Place-Based Leadership: Lesson-Drawing from Urban Governance Innovations in Bristol, UK», in Metropolitics, 2018. 


\section{"All of Us », la stratégie d'intégration sociale du maire}

Comme nous l'avons vu plus haut, l'engagement en faveur de l'intégration sociale a été renforcé par la nomination du premier adjoint au maire chargé de l'intégration sociale, de la mobilité sociale et de l'engagement communautaire. La vision de ce poste est inscrite dans deux documents: le programme d'intégration sociale intitulé «All of Us» («Tous ensemble $\left.{ }^{20} »\right)$, et le programme «Inclusive London» («Pour que Londres soit plus inclusive ${ }^{21} \gg$ ), le programme du maire en matière d'égalité. Comme nous l'avons souligné dans l'examen des stratégies antérieures de la mairie, ce dernier document s'inscrit en quelque sorte dans la continuité de l'effort précédemment porté sur l'égalité des chances. Le rapport de l'AGL qui l'accompagne décrit comment ces orientations aident l'AGL à remplir ses obligations en vertu de la loi de 2010 sur l'égalité22. Il démontre une approche qui se concentre sur les caractéristiques statutaires protégées telles qu'elles sont définies dans la loi, plutôt que spécifiquement sur l'immigration ou l'intégration des communautés immigrées au sens large, comme on peut le voir dans la seconde mouture du programme "London Enriched» («Enrichissons Londres»).

De la même manière, la stratégie d'intégration sociale se distingue par l'élaboration d'une définition de l'intégration sociale et par l'établissement de quatre éléments clés de l'action de l'AGL en matière d'intégration sociale: les relations humaines, promouvoir les expériences partagées; la participation, aider Londoniennes et Londoniens à devenir des citoyens actifs; l'égalité, s'attaquer aux obstacles et aux inégalités; les données, améliorer la base de données de Londres permettant de mesurer, d'évaluer et de partager les résultats sur l'état de l'intégration sociale.

Dans le modèle de l'AGL, les trois premières sections se recoupent pour former un triangle où «tous ces [facteurs] délimitent l'étendue et la qualité des interactions et des liens entre Londoniennes et Londoniens de différents milieux, se renforçant les uns les autres ${ }^{23}$ ».

Au sein de ce modèle, le programme "Tous ensemble» fait trois critiques notables à la politique en matière d'intégration sociale telle qu'elle fonctionne au Royaume-Uni aux niveaux local et national, et expose les moyens par lesquels cette politique cherche à combler les lacunes grâce à sa nouvelle définition de l'intégration sociale. Premièrement, les théories de l'intégration sociale mettent trop l'accent sur la quantité des rapports sociaux, au détriment de leur qualité, affirmant qu'« une société véritablement intégrée socialement ne se résume pas à de simples interactions. Elle est constituée d'individus qui construisent des relations riches, que ce soit en tant qu'amis, collègues ou concitoyens ${ }^{24} 》$. Deuxièmement, la stratégie souligne qu'un regard trop étroit porté sur l'aspect «racial» et l'ethnicité «met trop l'accent sur l'intégration entre personnes de nationalités et d'ethnies différentes, négligeant d'examiner d'autres aspects importants de la division sociale, tels l'âge, la classe sociale, le statut professionnel, la sexualité, le sexe et le handicap ${ }^{25}$ ». Troisièmement, la stratégie contient clairement dans son titre l'idée que l'intégration sociale est une responsabilité partagée,

20. Greater London Authority, «All of Us. The Mayor's Social Integration Strategy », 2018.

21. Greater London Authority, «Inclusive London. The Mayor's Equality, Diversity and Inclusion Strategy », 2018. Url: https:// www.london.gov.uk/sites/default/files/mayors-equality-diversityinclusion-strategy.pdf.

22. Greater London Authority, «Publication of Inclusive London: The Mayors Equality, Diversity and Inclusion Strategy », 2018. Url: https://www.london.gov.uk/sites/default/files/md2212_inclusive_ london_signed_pdf.

23. Greater London Authority, «All of Us. The Mayor's Social Integration Strategy », op. cit.

24. Ibid.

25. Ibid. 
affirmant que, sans cela, elle peut donner l'impression que «l'intégration sociale ne se joue que dans l'action des communautés ethniques ou religieuses spécifiques, alors qu'elle est au contraire précieuse pour nous tous, et ce de manières très diverses ${ }^{26}{ }$.

Ainsi, le programme «Tous ensemble» présente à la fois des éléments en rupture et en continuité avec les politiques précédentes. Cette stratégie s'ancre dans la continuité d'une approche fondée depuis longtemps sur l'égalité, et souhaitant d'ailleurs élargir cette approche afin d'inclure un éventail plus large de caractéristiques allant au-delà des seules caractéristiques protégées par la loi. Toutefois, elle met en évidence une tension en affirmant que la dépendance excessive à l'égard de la «race» et de l'ethnie conduit à ce que l'intégration ne soit perçue qu'au travers de ce seul prisme. La solution qu'elle propose consiste à la fois en un élargissement de la définition de l'intégration sociale et en une prise en compte de l'intégration comme un processus à double sens de responsabilités partagées, par le biais duquel toutes les communautés participent au processus d'intégration, et pas seulement les communautés individuelles. Elle intègre ainsi à la fois une réflexion issue de la recherche universitaire $^{27}$ et des institutions publiques ${ }^{28}$.

II faut cependant remarquer que cette approche pourrait mener à un affaiblissement de l'attention accordée aux migrations et aux immigrés dans le cadre de ce programme. Contrairement aux modèles américains sur lesquels Sunder Katwala et son équipe ${ }^{29}$ ont étayé leurs recommandations, le rôle de l'adjoint au maire et de ce programme n'est pas exclusivement centré sur les communautés immigrées. Ce qui ne veut pas dire que ce programme ne contient pas de références à un travail ciblé avec les immigré-es - y compris les initiatives sur lesquels nous allons nous pencher-, mais plutôt qu'en élargissant la définition de l'intégration sociale, il fixe son propre cadre fondé sur l'égalité, la participation et les échanges entre toutes sortes de groupes sociaux. Il fixe les contours du problème politique visé ${ }^{30}$ au-delà des questions de migration.

\section{L'« initiative pour la citoyenneté et l'intégration » («Citizenship and Integration Initiative ")}

La stratégie d'intégration sociale de Londres s'éloigne quelque peu du cadre de l'intégration des immigré-es pour s'orienter vers un positionnement plus large. Elle a suscité le développement de toute une série d'initiatives et réformes institutionnelles: réunir le LSMP, le MRAP et la promotion des questions de politique nationale (comme le plaidoyer contre la mesure intitulée «Droit de louer »); faciliter l'accès à la citoyenneté grâce à l'initiative pour la citoyenneté et l'intégration (CII); développer un centre de ressources en ligne pour soutenir les ressortissants de l'Espace économique européen (EEE); coordonner la contribution du parrainage communautaire au programme de réinstallation syrien.

La CIl et le LSMP, notamment, offrent des approches complémentaires en matière de prestation de services par l'AGL dans les domaines de l'intégration et de l'immigration. La Cll est un fonds commun créé pour soutenir le travail sur la citoyenneté et l'intégration à Londres. Il est soutenu à la fois par l'AGL, par le biais d'investissements dans son équipe d'intégration sociale, et par un certain nombre de fondations caritatives indépendantes, dont l'objectif est de collecter et de distribuer 1 million de livres sterling entre 2017 et 2022.

Le Cll se fonde ainsi sur une articulation entre pratique caritative et action publique - inhabituel dans le contexte britannique - et instaure un partenariat entre des associations et le gouvernement local. Jusqu'à présent, le programme a aménagé une répartition des tâches entre la société civile et l'AGL, ce qui lui a permis de se concentrer sur un certain nombre de priorités stratégiques - en particulier l'engagement civique, l'accès des jeunes Londonien-nes aux droits, et des projets axés sur les rapports sociaux et l'affirmation de l'identité.

Le rapport d'étape rédigé par l'organisation «Trust for London» («Croyons en Londres», 2018 ${ }^{31}$ ) montre en quoi le programme est, de par ses objectifs - suivre les orientations politiques définies par la mairie, fournir directement des services et tester de

26. Ibid.

27. Sarah Spencer, Katharine Charsely, « Conceptualising Integration: A Framework for Empirical Research, Taking Marriage Migration as a Case Study », in Comparative Migration Studies, vol. 4, $n^{\circ} 18,2016$.

28. Commission on Social Integration, «Social Integration: A Wake up Call », 2014. Url: https://the-challenge.org/impact/ reports/social-integration-commission/.

29. Sunder Katwala, Steve Ballinger, Will Somerville, op. cit. 30. Carol Bacchi, «Policy as Discourse : What Does it mean? Where Does it Get us?», in Discourse : Studies in the Cultural Politics of Education, vol. 21, n 1, 2000, pp. 45-57.

31. Trust for London, "Citizenship and Integration Initiative Interim Report », 2018. Url: https://www.trustforlondon.org.uk/ publications/citizenship-and-integration-initiative-interim-report/. 
nouveaux modèles de prestation et de coopération -, un programme de nature expérimentale qui cherche à s'appuyer sur le rôle de leader de l'AGL pour faciliter et promouvoir le changement. Le rapport d'apprentissage montre comment la Cll a été utilisée comme plateforme pour développer les efforts en matière de promotion des droits. Elle les a enrichis par la promotion du travail en partenariat pour définir des programmes d'aide publique, mais aussi de plaider pour un changement politique, et notamment la suppression des frais de naturalisation pour les jeunes Londonien-nes. Avec cette initiative, l'AGL semble se rapprocher bien davantage de la vision décrite par Sunder Katwala ${ }^{32}$ en mettant à profit le rôle du maire adjoint afin de promouvoir les priorités politiques, telle que la "lutte active en faveur de la citoyenneté». De la même manière, dans son étude du ministère américain de l'Immigration, Els de Graauw identifie le leadership et la promotion de partenariats comme les fonctions essentielles de ces administrations, plus encore que la prestation de services, nous montrant ainsi comment l'organisation de la CII pourrait contribuer à renforcer ces deux fonctions $^{33}$. Il s'agit ici de fonder sa crédibilité politique sur la base de pratiques éprouvées.

Précisons néanmoins que les rôles du LSMP et du MRAP sont définis très différemment par l'AGL. La stratégie d'intégration sociale indique que le LSMP est financé par une subvention du ministère de l'Intérieur censée réunir « différentes organisations afin d'examiner les questions de migration et leur impact sur Londres et ses communautés immigrées ${ }^{34}$ », tout en décrivant le MRAP une entité censée apporter des conseils «sur les problèmes et les défis auxquels font face les communautés de réfugié-es et d'immigré-es à Londres ». La conception de ces deux rôles au sein du programme est essentiellement passive. Là où la CII vise à façonner pro-activement la politique de l'AGL et la promotion exercée par le gouvernement national en favorisant la création de partenariats stratégiques, le LSMP et le MRAP sont définis par le programme comme des instances où se réunissent les différents partenaires afin de fournir un espace de consultation et d'interaction, mais sans s'approprier les orientations politiques ni les développer.

Cette passivité peut s'expliquer par la nature centralisée et verticale du financement du LSMP par le ministère de l'Intérieur, ce qui oblitère les liens avec les agences au niveau local ${ }^{35}$. Ceci contraste avec ce que l'on observe dans d'autres villes que Londres: d'autres SMP se sont forgés une identité plus autonome et suivent un programme plus large. Ainsi, le bureau pour l'immigration du Yorkshire complète son rôle d'animateur en cherchant à endosser un rôle d'orientation stratégique, notamment en apportant des données et des résultats issus de la recherche sur des projets ciblés. À Londres, le SMP n'a pas émergé comme un forum stratégique et reste une entité quelque peu périphérique dans l'architecture de l'AGL.

\section{Une conception large de l'intégration}

Dans leur examen de la gouvernance en matière d'immigration et d'intégration, Peter Scholten et Rinus Penninx indiquent que "plusieurs maires successifs de l'Autorité du Grand Londres se sont montrés particulièrement proactifs en matière d'intégration de la population immigrée ${ }^{36} \gg$. Cependant, comme on l'a vu plus haut, s'il est vrai que «les villes ayant une diversité importante, comme Londres, embrassent la diversité comme faisant partie de leur identité et comme point d'ancrage positif des politiques locales, parfois en contradiction avec leurs modèles nationaux respectifs ${ }^{37}$ », Londres n'a pas toujours considéré l'élaboration et la pratique des politiques d'intégration comme une priorité. Cette négligence s'explique en partie par la structure de gouvernance de Londres, dans laquelle les 33 arrondissements conservent de nombreuses compétences qui se recoupent avec l'intégration (santé publique, services sociaux, attribution de logements, sécurité communautaire et aussi, bien que dans une moindre mesure, éducation), et où l'image de la gouvernance en matière d'intégration se révèle beaucoup plus complexe et différenciée que la mise en avant du personnage central qu'est le maire pourrait laisser penser.

Pour autant, les administrations successives ont développé des approches particulières en matière

32. Sunder Katwala, Steve Ballinger, Will Somerville, op. cit. 33. Els de Graauw, « Rolling Out the Welcome Mat: State and City Immigrant Affairs Offices in the United States », in IdeAs, $n^{\circ} 6$, 2015. Url: ideas.revues.org/1293.

34. Commission on Social Integration, « Social Integration: A Wake up Call », op. cit.

35. Si cela se vérifiait lorsque la stratégie a été publiée, en 2018, le recrutement récent a élargi l'équipe au-delà de ce mandat, offrant peut-être une marge d'action supplémentaire, comme nous allons le voir.

36. Peter Scholten, Rinus Penninx, «The Multilevel Governance of Migration and Integration », in Blanca Garces-Mascarenas, Rinus Penninx, (dir.), Integration Processes and Policies in Europe, Londres, Springer, 2016, pp. 91-109.

37. Ibid. 
d'intégration - tantôt largement en accord avec la politique du gouvernement national (comme le programme Livingstone qui s'inspire du programme «New Labour» du parti travailliste en matière d'égalité), tantôt de manière apparemment plus désolidarisée. Ainsi, le plaidoyer de Khan sur les droits des citoyen-nes européen-nes et sur l'accès à la citoyenneté des jeunes Londonien-nes se distingue de la position du gouvernement May au niveau national. Le leadership, la fonction symbolique incarnés par le maire et l'importance de la communication ont renforcé l'impression d'un positionnement distinct de l'agenda national, donnant l'impression d'une mairie londonienne qui adopte une approche militante.

10 Dans le même temps, si le programme « Tous ensemble » est en synergie avec certains aspects des politiques nationales en matière d'intégration, il ne manque pas de les critiquer.

Dans le même temps, si le programme «Tous ensemble» est en synergie avec certains aspects des politiques nationales en matière d'intégration, il ne manque pas de les critiquer. Le programme s'éloigne d'une approche ouvertement axée sur l'immigration, pour adopter une définition plus large de l'intégration sociale englobant d'autres facteurs, telle l'intégration intergénérationnelle. Ce faisant, il permet certaines avancées politiques - notamment en promouvant le sens de la responsabilité partagée, présent dans une grande partie de la littérature académique et de l'élaboration des politiques, mais souvent absent dans la pratique. La formule «All of Us» définit clairement l'intégration sociale comme une voie à double sens identifiée dans les travaux académiques, évitant ainsi de faire porter la responsabilité de l'intégration au seul groupe d'immigré-es, et tentant de s'éloigner des conceptions idéologiques normatives souvent charriées par le terme d'«intégration », comme l'a montré Willem Schinkel en 2018, critiquant l'usage même du terme ${ }^{38}$. La définition de l'intégration privilégiée par cette stratégie, en mettant l'accent sur la qualité des rapports plutôt que sur la seule quantité - dans le domaine des relations humaines -, permet de faire émerger une conception plus large de l'intégration, une dynamique qui serait à la fois inscrite dans l'espace de la ville et dépendante des atouts (sociaux, économiques, etc.) locaux ${ }^{39}$. Elle se démarque ainsi d'une perspective politique fondée sur une conception de l'intégration perçue comme un «déficit» et proposant la seule élimination des obstacles à l'inclusion.

\section{La singularité du modèle londonien}

Cette approche dépasse une conception de l'inclusion exclusivement axée sur l'immigration, et ce d'une manière qui peut sembler surprenante, étant donné le rôle non négligeable que celle-ci joue dans la composition démographique de la ville. Le rôle plus limité du LSMP explique pourquoi les choix politiques se traduisent par une prestation de services moins axée sur l'immigration et ses impacts en tant que priorité politique générale. S'il est sans doute vrai que l'AGL a pris position sur un certain nombre de questions politiques - droits réciproques avec les citoyens de l'Union européenne, accès à la citoyenneté par le biais de la CII, défense de la position du maire sur la question de l'immigration en 2017 -, il n'en demeure pas moins que l'objectif principal de la stratégie d'intégration sociale est d'adopter une perspective plus large que celle de l'immigration.

Cette focale plus large de l'AGL pose la difficulté de désarticuler son action de celle du gouvernement central concernant le système d'immigration. Par ailleurs, elle n'est pas calibrée pour se concentrer sur le cadre temporel des «nouveaux venus», une problématique pourtant essentielle étant donnée la dynamique de «roulement» et de circulation qui caractérise une ville comme Londres. Grâce à l'initiative de la CII, l'AGL a développé une manière novatrice de réunir et de soutenir les partenaires issus de la société civile, mais aussi d'agir de manière co-productive, à la fois par la division du travail qui résulte de ces partenariats et en combinant les sources de financement public avec des initiatives bénévoles indépendantes. Toutefois, comme l'indique le rapport faisant le bilan du programme "Trust for London», l'aménagement de ce modèle s'est heurté à des difficultés, certaines initiatives ayant mis plus de temps à se mettre en place. Le rapport constate que la CII a

38. Willem Schinkel, «Against "Immigrant Integration": For an End to Neocolonial Knowledge Production », in CMS, vol. 6, $n^{\circ} 31$, 2018.

39. Michael Lyons, «The Lyons Inquiry into Local Government », 2007. Url:https://www.webarchive.org.uk/wayback/ archive/20070428120000/http ://www.lyonsinquiry.org.uk/docs/ final-complete.pdf. 
bien été en mesure d'apporter des suggestions politiques à la stratégie d'intégration sociale, mais il est frappant de constater que les initiatives de la CII se concentrent de manière beaucoup plus ciblée sur les questions d'immigration et de citoyenneté - accès à la citoyenneté pour les jeunes Londonien-nes, conseil en matière d'immigration, cérémonies de citoyenneté, inscription sur les listes électorales -, ce qui semble indiquer un écart entre des ambitions générales visant à dépasser la question de l'immigration et une orientation plus ciblée sur les immigré-es au sein de la Cll.

En établissant un modèle de leadership inclusif, axé sur l'ancrage dans un lieu donné, Robin Hambleton identifie pour les dirigeants cinq domaines de légitimation se chevauchant: le politique, le communautaire, la gestion, les syndicats et organisations représentatives d'entrepreneurs ${ }^{40}$. En ce qui concerne l'AGL, le maire et son adjoint ont très certainement joué un rôle de légitimation politique; la mise en œuvre de projet et sa gestion des partenariats ont donné une légitimité en matière de gestion; les efforts de renforcement du leadership communautaire menés par la Cll ont renforcé sa légitimité communautaire. Hambleton décrit les croisements entre ces domaines comme des «zones d'innovation», et c'est bien dans ces domaines qu'il reste à voir dans quelle mesure l'AGL pourra faire coïncider ses différents champs d'activité.

\section{Conclusion}

Le rôle de l'AGL en matière d'intégration sociale a été cactérisé par sa singularité. L'AGL a assuré une continuité entre les différents mandats des maires tout en évoluant constamment. Dans le contexte de cette singularité londonienne au Royaume-Uni, tant sur le plan de la gouvernance que sur le plan démographique, l'AGL a mis en place ses propres outils d'élaboration et de compréhension de l'intégration sociale par le biais du programme "Tous ensemble». Le programme et son approche restent néanmoins fortement marqués par l'histoire de la politique d'intégration sociale mise en œuvre à partir de 2000, une histoire faite de la sédimentation des approches des différentes administrations municipales et de la façon dont elles ont pu adhérer ou s'éloigner des tendances dominantes au niveau national.

Le programme «Tous ensemble» met en évidence une tension centrale dans l'élaboration des politiques et la pratique en matière d'intégration au niveau des collectivités locales au Royaume-Uni. En dépit de la nature locale des processus d'intégration, les rôles des collectivités territoriales en la matière ne sont pas toujours clairement définis, tandis que cette question a traditionnellement été relativement peu importante. Afin de pallier cette tension, le programme se fonde sur une conception large de l'intégration sociale, reposant sur une approche en matière d'égalité, en élargissant cette approche pour intégrer d'autres catégories sociales telles que le genre ou le handicap. Ce faisant, elle incorpore certains principes fondamentaux des résultats de la recherche en matière d'intégration, à savoir la nécessité de reconnaître que l'intégration est une dynamique à double sens, et d'établir un lien avec la conception de l'administration locale en tant que gestionnaire d'un espace de vie où se côtoient différentes communautés, construisant son action sur sa capacité d'orientation stratégique et sa capacité à générer des partenariats sur la base d'objectifs partagés.

Cette conception plus large porte cependant en elle le risque qu'une telle stratégie perde de vue l'un des sujets politiquement les plus délicats, mais aussi les plus cruciaux: celui de la migration récente, diminuant ainsi la capacité du gouvernement local à s'engager politiquement et à apporter des solutions crédibles à une problématique clé. Sur cet aspect, l'approche de l'AGL en matière d'intégration sociale se démarque des orientations exprimées par le maire, notamment dans le cadre de la campagne «Londres est ouverte», lesquelles mettent l'accent sur l'immigration et les nouveaux arrivants, situant cet engagement dans le contexte de l'histoire de Londres. L'approche de l'intégration sociale de l'AGL brouille quelque peu le message de ce discours. Ce faisant, elle ouvre aussi de nouvelles voies qui pourront permettre à Londres d'évoluer en rattachant son approche originale des rapports sociaux, de la participation et de la responsabilité partagée à une approche du leadership ancrée dans un contexte donné. Ainsi, elle sera à même d'utiliser l'expérience accumulée au fil de l'histoire de Londres, de ses engagements politiques et de son leadership politique, pour développer le programme d'intégration sociale de la ville.

Article traduit de l'anglais par Alexandre Pateau.

40. Robin Hambleton, op. cit. 